\title{
A point mutation in the putative ATP binding site of the pseudorabies virus US3 protein kinase prevents Bad phosphorylation and cell survival following apoptosis induction
}

\author{
Matthias Deruelle, Kristin Geenen, Hans J. Nauwynck, Herman W. Favoreel* \\ Department of Virology, Parasitology, and Immunology, Faculty of Veterinary Medicine, Ghent University, \\ Salisburylaan 133, 9820 Merelbeke, Belgium \\ Received 12 January 2007; received in revised form 5 April 2007; accepted 5 April 2007 \\ Available online 11 May 2007
}

\begin{abstract}
The multifunctional US3 protein kinase is conserved among alphaherpesviruses. Like the herpes simplex virus US3 protein kinase, the pseudorabies virus (PRV) US3 protein confers resistance against apoptosis. In the current report, we introduced a point mutation in the putative ATP binding site of the PRV US3 protein kinase. We found that, in contrast to the wild type PRV US3, the point-mutated PRV US3 does not protect cells from apoptosis induced by PRV infection or staurosporine treatment. In addition, we found that the presence of wild type PRV US3, but not of the point-mutated PRV US3, results in phosphorylation of the pro-apoptotic Bad protein in PRV-infected ST and HEp-2 cells. In PRV-infected ST cells, but not in HEp-2 cells, an additional, US3- and phosphorylation-independent alteration of Bad could be observed. These results suggest that the kinase activity of the US3 protein of PRV is crucial to protect cells from apoptotic cell death during infection, at least partly by leading to phosphorylation of the pro-apoptotic Bad protein.
\end{abstract}

(C) 2007 Elsevier B.V. All rights reserved.

Keywords: Pseudorabies virus; US3 protein kinase; Apoptosis; Bad phosphorylation

\section{Introduction}

Alphaherpesviruses comprise a closely related subfamily of the herpesviruses that cause relatively mild, but sometimes devastating disease, including encephalitis. The viral US3 serine/threonine protein kinase is conserved among alphaherpesviruses and is a multifunctional protein. The US3 kinase orthologues of herpes simplex virus (HSV) and, recently, of the porcine pseudorabies virus (PRV), have been shown to suppress apoptosis induced by alphaherpesvirus infection or by external stimuli (e.g. Leopardi et al., 1997; Ogg et al., 2004; Geenen et al., 2005). The US3 kinases of HSV type 1 (HSV1), PRV and Marek's disease virus have been demonstrated to be implicated in viral egress from the nucleus (Wagenaar et al., 1995; Klupp et al., 2001; Reynolds et al., 2002; Schumacher et al., 2005). Recently, we and others have shown that the US3 kinase of different alphaherpesviruses induces alterations in the

\footnotetext{
* Corresponding author. Tel.: +32 926473 74; fax: +329264 7495 .

E-mail address: Herman.Favoreel@UGent.be (H.W. Favoreel).
}

actin cytoskeleton. These actin rearrangements consist of the disassembly of actin stress fibers (Murata et al., 2000; Van Minnebruggen et al., 2003; Schumacher et al., 2005), and the formation of long, branched cellular projections (Calton et al., 2004; Favoreel et al., 2005), and are associated with enhanced intercellular virus transmission (Favoreel et al., 2005). Furthermore, the US3 kinase has been reported to be involved in downregulating the major histocompatibility complex class I (MHC I) expression in varicella-zoster virus (VZV)-infected cells (Abendroth et al., 2001), in the HSV-induced inactivation of cytotoxic T-lymphocytes (CTLs) (Sloan et al., 2003) and in the reduced sensitivity of HSV-1 and VZV-infected cells to interferon (Piroozmand et al., 2004; Schaap et al., 2005).

How US3 exerts its different functions is far from fully understood. Since the US3 protein possesses serine/threonine kinase activity, an important first step to understand the mechanism of US3-induced effects, is to analyze whether they rely on the kinase activity of the protein. Kinase activity has recently been shown to be important for the function of US3 during nuclear egress, since a point-mutated, kinase-impaired US3 protein of HSV-1 was found to be unable to complement the reduced 
efficiency in virus egress from the nucleus of a US3null virus (Ryckman and Roller, 2004). Kinase activity of HSV-1 US3 has also been found to be important for suppressing apoptosis induced by overexpression of pro-apoptotic Bcl-2 family members (Ogg et al., 2004). Whether kinase activity of US3 is equally important for protecting cells from apoptosis during alphaherpesvirus infection or during apoptosis induction by other exogenous stimuli is not known.

In the current report, we investigated whether mutation of a conserved lysine residue in the putative ATP binding domain of US3 had an effect on its anti-apoptotic properties during infection or treatment with staurosporine. We report that this mutation indeed disrupts the ability of PRV US3 to protect cells from apoptosis. In addition, we show that intact kinase activity of PRV US3 results in phosphorylation of the pro-apoptotic Bad protein during PRV infection and that other, US3- and phosphorylationindependent and cell type-specific Bad alterations may occur in PRV-infected cells.

\section{Methods}

\subsection{Construction of point-mutated US3}

Construction was done, essentially as described before for HSV US3, by the introduction of a point mutation in a conserved lysine residue that is critical for ATP binding (Ryckman and Roller, 2004). Mutation consisted of a substitution of lysine 136 in the PRV US3 ORF by glutamine and was introduced by PCR using pfx polymerase. Primers to introduce the mutation were US3mutF (ACGGTGGTGCTGCAGGTGGGCCAGA) and US3mutR (reverse complement of US3mutF). In a first step, the point-mutated $5^{\prime}$ region of US3 was generated and PCR amplified from a plasmid containing the entire US3 ORF under control of the CMV promotor (pKG1, Geenen et al., 2005) using a T7 primer (Invitrogen) (T7 promoter is located $5^{\prime}$ of US3 in pKG1) and US3mutR. The point-mutated $3^{\prime}$ region of US3 was generated and PCR amplified from pKG1 using US3mutF and a BGH primer (Invitrogen, Groningen, The Netherlands) (BGH promoter is located $3^{\prime}$ of US3 in pKG1). In a second step, the entire point-mutated US3 ORF was generated and PCR amplified using $\mathrm{T} 7$ and $\mathrm{BGH}$ primers, using a mixture of both former PCR products as targets. The resulting PCR product was checked for the presence of the mutation and the absence of other mutations by restriction digestion (mutation introduces an extra PstI site) and sequencing. The point-mutated US3 ORF was digested with HindIII and NotI, and the resulting $1366 \mathrm{bp}$ fragment, containing the entire point-mutated US3 ORF, was cloned in a HindIII-NotI-digested pKG1 backbone, resulting in pHF61.

\subsection{Viruses, cells and reagents}

Swine testicle (ST) cells were used and cultured as described before (Geenen et al., 2005). Human laryngeal epidermoid carcinoma HEp-2 cells were cultured in Eagle's minimal essential medium (MEM) with $10 \%$ foetal calf serum (FCS), $100 \mathrm{U} / \mathrm{ml}$ penicillin, $0.1 \mathrm{mg} / \mathrm{ml}$ streptomycin, $0.3 \mathrm{mg} / \mathrm{ml}$ glutamine and
$1 \times$ MEM non-essential amino acids. Wild type and isogenic US3null PRV NIA-3 viruses were used. The latter has been described and characterized earlier and is an NIA3 wild type derived mutant that was constructed by insertion of a palindromic 20-mer oligonucleotide, containing TAG translational stop codons in all reading frames, in the $5^{\prime}$ part of the US3 open reading frame (ORF). Insertion of this oligonucleotide in any protein-encoding gene, in any orientation and in any reading frame results in premature translational termination (de Wind et al., 1990; van Zijl et al., 1988, 1990). Plasmid pcDNA3.1D/V5-His ${ }^{\circledR} / \mathrm{lacZ}$ (pLacZ/V5), anti-V5 antibodies, FITC-labeled goat anti-mouse and goat anti-rabbit antibodies, Texas red-labeled goat anti-mouse antibodies, Lipofectamine ${ }^{\mathrm{TM}}$ and Dynabeads ${ }^{\circledR}$ Protein A were from Invitrogen (Carlsbad, California, USA). US3-specific mouse monoclonal antibodies were kindly provided by LeighAnne Olsen and Lynn Enquist (Princeton University, USA). Rabbit antibodies directed against active caspase-3 and human/mouse Bad were from R\&D Systems Inc. (Minneapolis, MN). HRP-conjugated secondary goat anti-rabbit and goat anti-mouse antibodies were purchased from Dako Cytomation (Denmark), calf intestinal alkaline phosphatase (CIP) and corresponding reaction buffer from New England BioLabs (Ipswich, MA) and both Hybond-P PVDF membrane and enhanced chemiluminescence (ECL) reagents from Amersham Biosciences (GE Healthcare, Buckinghamshire, UK). Staurosporine, BSA and both rabbit anti-actin and mouse anti-phospothreonine antibodies were from Sigma Chemical Company (St. Louis, Missouri). Hydrogen peroxide and citric acid were purchased from VWR International Inc. (San Diego, California, USA).

\subsection{Transfections}

Plasmids pKG1, pHF61 or control plasmid pLacZ were transiently transfected in cells grown to $60-70 \%$ confluency using Lipofectamine ${ }^{\mathrm{TM}}$ according to the manufacturer's instructions (Invitrogen), and used at $24 \mathrm{~h}$ post-transfection.

\subsection{Apoptosis assays}

Apoptosis was induced by infection of cells with US3null PRV (m.o.i. of 10 ) for $24 \mathrm{~h}$ or by the addition of $1.5 \mu \mathrm{M}$ staurosporine for $12 \mathrm{~h}$, followed by immunofluorescent detection of the presence of active caspase-3 (using caspase-3-specific rabbit antibodies and FITC-labeled goat anti-rabbit antibodies) and US3 (using US3-specific mouse antibodies and Texas red-labeled goat anti-mouse antibodies), and analysis by fluorescence and confocal microscopy, all as described before (Geenen et al., 2005).

\subsection{Detection of Bad phosphorylation and actin (loading control)}

Phosphorylation of Bad was assessed, essentially as described before (Cartier et al., 2003). In brief, both ST and HEp-2 cells were either not transfected or transfected with pKG1 or pHF61. At $24 \mathrm{~h}$ post-transfection, cells were either 
mock-infected or infected with WT PRV or US3null PRV. All infections were done at an m.o.i. of 10 and in a total volume of $1 \mathrm{ml}$ culture medium. At $24 \mathrm{~h}$ post-inoculation, cells were collected on ice using a rubber policeman, washed in TNE buffer (50 mM Tris, $150 \mathrm{mM} \mathrm{NaCl}, 1 \mathrm{mM}$ EDTA, pH 6.8) and lysed in TNE lysis buffer (TNE with $1 \%$ Triton-X 100, $1 \mathrm{mM} \mathrm{Na} \mathrm{VO}_{4}, 10 \mathrm{mM} \mathrm{NaF}$, protease inhibitor cocktail) for $1 \mathrm{~h}$ at $4{ }^{\circ} \mathrm{C}$. Cell lysates were fractionated on a $15 \%$ polyacrylamide gel by SDS-PAGE and then transferred to a Hybond-P PVDF membrane. After blotting, the membranes were blocked in 5\% non-fat dry milk in $0.1 \%$ PBS/Tween-20 for $1 \mathrm{~h}$ at room temperature (RT), and incubated with affinity purified rabbit anti-human/mouse Bad overnight at $4{ }^{\circ} \mathrm{C}$. Following incubation with HRP-conjugated secondary goat anti-rabbit antibodies, blots were developed by ECL. After development, blots were washed and incubated with rabbit anti-actin. Following incubation with HRP-conjugated secondary goat anti-rabbit antibodies, a second ECL was performed. All incubation steps were done in blocking buffer.

\subsection{Detection of threonine-phosphorylated proteins and actin (loading control)}

The same ST cell lysates as mentioned in Section 2.5 were loaded on a $12 \%$ polyacrylamide gel. After Western blotting, the blot was blocked in 5\% BSA in $0.2 \%$ TBS/Tween-20 for $1 \mathrm{~h}$ at RT, and then washed overnight. Following incubation with mouse anti-phosphothreonine and HRP-conjugated secondary goat anti-mouse antibodies, the blot was developed by ECL. All incubation steps were done in blocking buffer. After development, the blot was washed and then treated with $15 \%$ hydrogen peroxide during $30 \mathrm{~min}$ at RT to remove the remaining HRP signal. Finally, actin was detected as described in Section 2.5.

\subsection{In vitro phosphatase treatment}

ST cells were either mock-infected or infected with WT PRV at an m.o.i. of 10 in a total volume of $1 \mathrm{ml}$ culture medium. At $24 \mathrm{~h}$ post-inoculation, cells were collected and washed as described in Section 2.5. Following cell lysis in TNE lysis buffer as described in 2.5 but without phosphatase inhibitors, Bad was immunoprecipitated using Dynabeads ${ }^{\circledR}$ Protein A according to the manufacturer's instructions (Invitrogen). Before elution, the bead-antibody-Bad complexes were treated with either 0 or 10 units CIP for $1 \mathrm{~h}$ at $37^{\circ} \mathrm{C}$. Finally, immunoprecipitated Bad was eluted in $0.1 \mathrm{M}$ citrate $\mathrm{pH} 3.1$ and $2 \times$ SDS-PAGE loading buffer was added. SDS-PAGE, Western blotting and Bad detection were performed as described in Section 2.5.

\subsection{Detection of US3 expression levels in transfected cells}

ST cells were either not transfected or transfected with pKG1 or pHF61. At $24 \mathrm{~h}$ post-transfection, cells were harvested by trypsinisation and washed in PBS. Cells were fixed in $3 \%$ paraformaldehyde for $10 \mathrm{~min}$ at RT, followed by permeabilization using $0.1 \%$ Triton-X 100 for 2 min at RT. Both the primary and secondary incubation, using mouse anti-US3 and
FITC-conjugated goat anti-mouse antibodies, respectively, were performed at $37^{\circ} \mathrm{C}$ for $1 \mathrm{~h}$. All antibody dilutions were made in PBS. Finally, fluorescence intensity of the cells was analyzed by flow cytometry (FACSCanto, Beckton-Dickinson Biosciences, San Jose, California), analyzing 10,000 cells.

\section{Results}

\subsection{Construction of a eukaryotic expression vector encoding a PRV US3 protein containing a point mutation in its putative ATP binding domain}

The US3 protein kinase of PRV exists as two isoforms, a long and a short isoform, with the long isoform only differing from the short isoform by the presence of an $\mathrm{N}$-terminal mitochondrial localisation signal (Van Minnebruggen et al., 2003; Calton et al., 2004). Interestingly, the long isoform, although the less abundant isoform in infected cells, appears to contain the strongest capacity to suppress apoptosis (Geenen et al., 2005).

A eukaryotic expression vector encoding the long isoform of PRV US3 (pKG1) was used as a template to create the mutated variant of US3. A plasmid derived from pKG1 and encoding a kinase-impaired recombinant PRV US3 protein (pHF61) was constructed, principally as done before for HSV-1 US3 (Ryckman and Roller, 2004), by point mutation of a conserved lysine residue in the US3 ORF that is critical for ATP binding (Leung-Tack et al., 1994; Ryckman and Roller, 2004). Correct mutagenesis was checked by restriction enzyme digestion (additional PstI site) and by sequencing.

Using flow cytometry, we confirmed that both wild type and point-mutated US3 show similar protein expression levels (Fig. 1).

\subsection{Intact kinase activity of PRV US3 is necessary for exerting its anti-apoptotic effects}

Earlier, we have found that PRV US3, like its HSV-1 orthologue, is able to suppress apoptosis induced by virus infection or staurosporine (Leopardi et al., 1997; Geenen et al., 2005).

To investigate a possible involvement of the kinase activity of US3 in apoptosis induced by an alphaherpesvirus infection or staurosporine addition, ST cells were transiently transfected with either pLacZ (a control plasmid), pKG1 (encoding wild type US3), or pHF61 (encoding point-mutated US3), and, at $24 \mathrm{~h}$ post-transfection, subjected to apoptosis-inducing stimuli: infection with US3null PRV (Fig. 2A) or addition of $1.5 \mu \mathrm{M}$ stauroporine (Fig. 2B). Transfected cells were subsequently analyzed for the activation of caspase-3. Both Fig. 2A and B show relative percentages of apoptosis, compared to the percentage of apoptosis observed in cells transfected with the control plasmid and infected with US3null PRV (Fig. 2A) or treated with staurosporine (Fig. 2B), which were set to 100\%. Absolute percentages of apoptosis observed in cells transfected with the control plasmid and subsequently infected with US3null PRV or treated with staurosporine were approximately $30-40 \%$.

Infection of lacZ-transfected cells with wild type instead of US3null PRV led to a decrease in the relative percentage of 

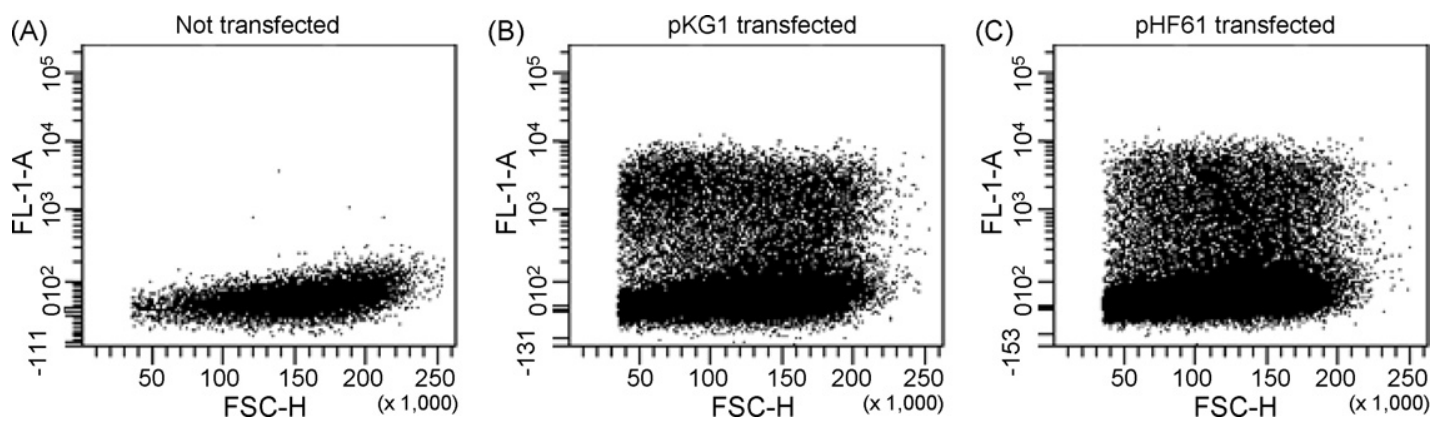

Fig. 1. Wild type (B) and point-mutated US3 (C) show similar protein expression levels. ST cells were either not transfected (A) or transfected with pKG1 (B) or pHF61 (C). At $24 \mathrm{~h}$ post-transfection, cells were harvested, fixed and permeabilized. Following an immunofluorescence staining using mouse anti-US3 and FITC-conjugated goat anti-mouse antibodies, fluorescence intensity of the cells was analyzed by flow cytometry, analyzing 10,000 cells. $X$-axis shows cell size, $Y$-axis fluorescence intensity. The latter corresponds with US3 protein expression levels.

apoptotic cells from 100 to $68.6 \pm 4.1 \%$. Similarly, transfection with wild type US3 instead of lacZ and subsequent infection with US3null PRV led to a decrease in the relative percentage of apoptotic cells from 100 to $56.3 \pm 3.1 \%$, all in agreement with earlier observations (Geenen et al., 2005). In contrast, cells transfected with the point-mutated US3 were not able to withstand apoptosis induced by US3null PRV (relative percentage of apoptosis: $100.1 \pm 1.3 \%$ ).

Comparable results were obtained using staurosporine to induce apoptosis instead of US3null PRV and are illustrated in Fig. 2B. Again, cells transfected with wild type US3, but not with mutant US3, were able to suppress apoptosis.

Together, these results show that a point mutation in the putative ATP binding site of the US3 protein of PRV abolishes its anti-apoptotic function during infection and staurosporine treatment.

\subsection{Intact kinase activity of PRV US3 results in phosphorylation of Bad}

For HSV-1, it has been shown that the US3 protein kinase phosphorylates, and thereby inactivates, the pro-apoptotic $\mathrm{Bcl}-2$ family member Bad, which may partly explain the mechanism of the anti-apoptotic activity of this protein (Cartier et al., 2003; Kato et al., 2005). We determined whether PRV US3 also induces phosphorylation of the pro-apoptotic Bad. Since it has been shown that PRV US3 displays anti-apoptotic activity in both ST (Geenen et al., 2005) and HEp-2 cells (Ogg et al., 2004), we investigated a possible PRV US3-mediated Bad phosphorylation in both cell types. Phosphorylation of Bad in PRV-infected HEp-2 and ST cells was assessed by detection of a band shift in Western blot analysis, as described before (Cartier et al., 2003).

Western blot analysis on HEp-2 (Fig. 3A) and ST cells (Fig. 3C) indeed revealed a shift of the Bad protein to a higher - likely phosphorylated - band during infection with WT PRV, compared to mock-infected cells. ST cells showed higher basal levels of phosphorylated Bad than HEp-2 cells (compare band patterns of mock-infected cells in Fig. 3A and C). Treatment of immunoprecipitated Bad from mock- or WT PRV-infected ST and HEp-2 cells with phosphatase confirmed that the upper Bad bands represent phosphorylated Bad (Fig. 4). Infection of ST cells with wild type PRV resulted in Bad phosphorylation, whereas infection with US3null PRV did not (Fig. 3A and C, lanes 2 and 3), indicating that Bad phosphorylation is US3mediated. Transfection of wild type US3 prior to infection with US3null virus restored Bad phosphorylation, whereas transfection with point-mutated US3 did not (Fig. 3A and C, lanes 4
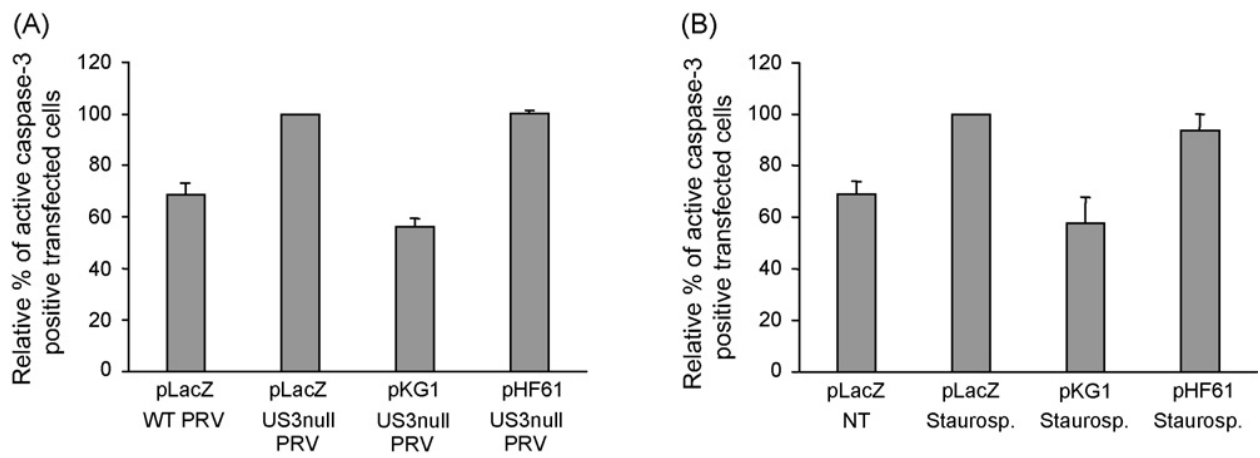

Fig. 2. A US3 recombinant carrying a point mutation in the putative ATP binding site is unable to protect cells against apoptosis induced by US3null PRV (A) or staurosporine (B). ST cells were transfected with pLacZ (control), pKG1 (wild type US3) or pHF61 (point-mutated US3). At 24h post-transfection, cells were inoculated with wild type or US3null PRV (A) or cells were left untreated (NT) or treated with $1.5 \mu \mathrm{M}$ staurosporine (B). Afterwards, transfected cells were visualised with anti-V5 antibodies (pLacZ) or anti-US3 antibodies (pKG1 and pHF61) and apoptotic cells were visualised by anti-active caspase-3 antibodies. Percentages given are relative percentages compared to the number of apoptotic cells in pLacZ-transfected cells infected with US3null PRV (A) or treated with staurosporine (B) (each set to 100\%). Only the US3 or control transfected cells were considered in the calculations. Data represent mean \pm S.D. of three independent assays. 


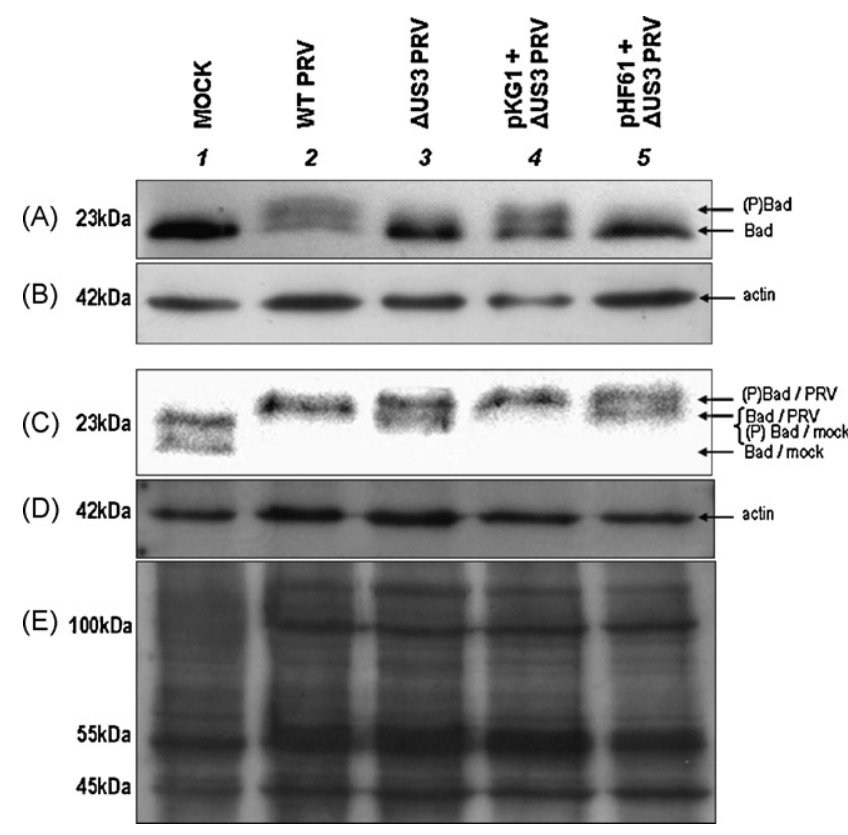

Fig. 3. Intact kinase activity of PRV US3 results in phosphorylation of the pro-apoptotic Bad protein in PRV-infected cells. WT PRV US3 induces Bad phosphorylation in PRV-infected HEp-2 (A and B) and ST-cells (C-E). ST and HEp-2 cells were either not transfected (lanes 1-3) or transfected with wild type (pKG1; lane 4) or mutant US3 (pHF61; lane 5). Twenty-four hours later, cells were either mock-infected (lane 1), or infected with WT PRV (lane 2) or US3null PRV (lanes 3 to 5). At $24 \mathrm{~h}$ post-inoculation, cells were collected and lysed for $1 \mathrm{~h}$ at $4{ }^{\circ} \mathrm{C}$. Total cell lysates were separated by SDS-PAGE followed by Western blot analysis with a Bad-specific antibody (A and C), an actin-specific antibody (B and D), or a phosphothreonine-specific antibody (E). Phosphorylated and unphosphorylated Bad in $\mathrm{A}$ and $\mathrm{C}$ are indicated by arrows. Panels $\mathrm{B}$ and D represent the actin loading controls. Panel E shows that the lack of Bad phosphorylation in the US3null PRV-infected cells is not due to a general lack of serine/threonine phosphorylation in these cells.

and 5). Thus, intact kinase activity of PRV US3 results in phosphorylation of Bad in PRV-infected cells. These data indicate that phosphorylation, and thereby inactivation, of Bad may be of importance in explaining the anti-apoptotic effect of US3. In addition, for each condition the total amount of threonine-

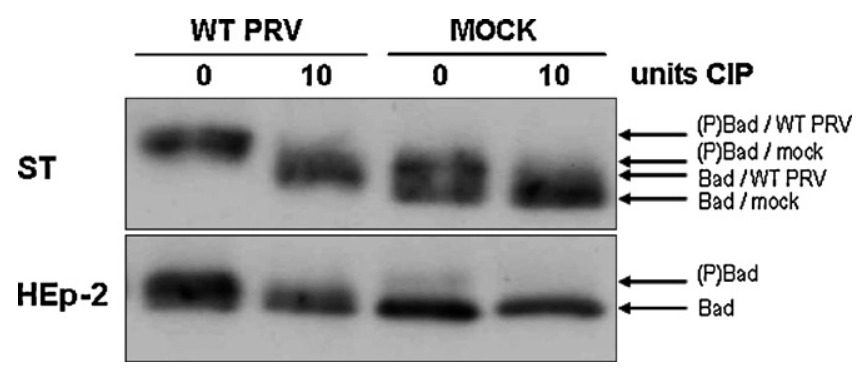

Fig. 4. Phosphatase treatment of immunoprecipitated Bad from mock- and WT PRV-infected ST (upper panel) and HEp-2 (lower panel) cells confirms that the upper Bad band represents phosphorylated Bad. Moreover, in PRV-infected ST cells, but not in HEp-2 cells, an additional, US3- and phosphorylationindependent alteration of Bad could be observed. At $24 \mathrm{~h}$ post-inoculation, both mock and WT PRV-infected ST and HEp-2 cells were collected. Following cell lysis, Bad was immunoprecipitated and then treated with either 0 or 10 units $\mathrm{CIP}$ for $1 \mathrm{~h}$ at $37^{\circ} \mathrm{C}$. Finally, immunoprecipitated Bad was eluted in $0.1 \mathrm{M}$ citrate $\mathrm{pH} 3.1$ and samples were separated by SDS-PAGE, followed by Western blot analysis using a Bad-specific polyclonal antibody. phosphorylated proteins was visualised on the ST blot, which confirmed that the lack of Bad phosphorylation in the US3null PRV-infected cells is not due to a general lack of serine/threonine phosphorylation in these cells (Fig. 3E). Proteins are indeed phosphorylated to similar levels, regardless whether cells are infected with wild type or US3null PRV, or whether cells are transfected with wild type or mutant US3 prior to US3null PRV infection, although, as expected, the phosphorylation pattern in infected cells differs from that in mock-infected cells (Fig. 3E).

Curiously, Bad migrated slower in WT and US3null PRVinfected ST cells compared to mock-infected ST cells, which was not observed in HEp-2 cells (Figs. 3A and C and 4). Interestingly, the slower migration was not phosphorylation-mediated, since phosphatase treatment did not shift the lower band in PRVinfected ST cells down to the level of the lower Bad band in mock-infected cells (Fig. 4). This indicates that there are US3and phoshorylation-independent alterations in Bad mobility in PRV-infected ST cells but not in HEp-2 cells.

\section{Discussion}

In the current report, we show that a point mutation in the putative ATP binding site of the PRV US3 kinase destroys the anti-apoptotic activity of this protein during PRV infection and staurosporine treatment. In addition, we found that the intact, but not the mutated, US3 protein of PRV results in phosphorylation of the pro-apoptotic protein Bad in PRV-infected ST and HEp2 cells. Moreover, we found that in PRV-infected ST cells, but not in HEp-2 cells, an additional, US3- and phosphorylationindependent alteration of Bad occurs.

Our finding that a kinase-intact PRV US3 results in phosphorylation of the pro-apoptotic protein Bad in PRV-infected cells is in line with reports on the potential anti-apoptotic effect of HSV$1 \mathrm{US} 3$, which has been reported to phosphorylate Bad (Cartier et al., 2003; Kato et al., 2005). These data indicate that phosphorylation, and thereby inactivation, of Bad may be of importance in explaining the anti-apoptotic effect of US3. This may also fit in our earlier observation that the mitochondria-located long isoform of US3 shows more pronounced anti-apoptotic activity than the short isoform that does not migrate to mitochondria (Geenen et al., 2005). For the cellular protein kinase A, it has been shown before that its mitochondrial localisation is of crucial importance for efficient phosphorylation of Bad, thereby preventing apoptosis (Harada et al., 1999). Of particular interest in this context is that HSV-1 US3 has been reported to functionally overlap protein kinase A to prevent apoptosis (Benetti and Roizman, 2004) and has very recently been shown to cofractionate with mitochondria (Poon et al., 2006). However, it is important to also point out that it has been shown for HSV-1 that US3 still displays anti-apoptotic activity in cells that express a mutant form of Bad that cannot be phosphorylated on the regulatory serines at positions 112,136 , and 155 , indicating that US3 may contain additional anti-apoptotic activities, other than Bad phosphorylation at these specific sites (Benetti et al., 2003; Ogg et al., 2004).

We also noticed that US3null PRV-infected cells expressing wild type US3 in trans show a somewhat better protection 
against apoptosis than wild type PRV-infected cells (US3 in cis) (Fig. 2), which confirms our earlier findings (Geenen et al., 2005). This is probably due to the higher expression levels of US3 in transfected cells than in infected cells, leading to a better protection against apoptosis. Interestingly, we observed a US3- and phosphorylation-independent shift in Bad mobility in Western blot in PRV-infected ST cells, which was not present in PRV-infected HEp-2 cells (Figs. 3A and C and 4). Further experiments will be needed to determine whether this cell typedependent alteration in Bad mobility reflects a species-specific difference in PRV-mediated Bad alteration, which viral proteins may be involved, and what the consequences may be for virus replication and apoptotic cell death.

In conclusion, we found that a point mutation in the putative ATP binding site of PRV US3 disrupts the ability of this protein to protect cells from apoptosis during infection and staurosporine treatment and prevents Bad phoshorylation in PRV-infected ST and HEp-2 cells, and that additional, cell type-dependent and US3- and phosphorylation-independent alterations in Bad may occur in PRV-infected cells.

\section{Acknowledgments}

The authors would like to thank LeighAnne Olsen and Lynn Enquist (Princeton University, USA) for US3-specific antibodies, the ID-DLO (Lelystad, the Netherlands) for NIA-3 PRV strains, and Nele Dennequin, Carine Boonen, Lieve Sys and Chantal Vanmaercke for excellent technical assistance. This research was supported by a cooperative research action fund of the Research Council of Ghent University and a research grant from the Research Foundation-Flanders (FWO-Vlaanderen), grant nr. G.0196.06.

\section{References}

Abendroth, A., Lin, I., Slobedman, B., Ploegh, H., Arvin, A.M., 2001. VaricellaZoster virus retains major histocompatibility complex class I proteins in the golgi compartment of infected cells. J. Virol. 75, 4878-4888.

Benetti, L., Munger, J., Roizman, B., 2003. The herpes simplex virus 1 US3 protein kinase blocks caspase-dependent double cleavage and activation of the proapoptotic protein Bad. J. Virol. 77, 6567-6573.

Benetti, L., Roizman B,., 2004. Herpes simplex virus protein kinase US3 activates and functionally overlaps protein kinase A to block apoptosis. Proc. Natl. Acad. Sci. U.S.A. 101, 9411-9416.

Calton, C.M., Randall, J.A., Adkins, M.W., Banfield, B.W., 2004. The pseudorabies virus serine/threonine kinase Us3 contains mitochondrial, nuclear and membrane localization signals. Virus Genes 29, 131-145.

Cartier, A., Komai, T., Masucci, M.G., 2003. The Us3 protein kinase of herpes simplex virus 1 blocks apoptosis and induces phosporylation of the Bcl-2 family member Bad. Exp. Cell. Res. 291, 242-250.

de Wind, N., Zijderveld, A., Glazenburg, K., Gielkens, A., Berns, A., 1990. Linker insertion mutagenesis of herpesviruses: inactivation of single genes within the Us region of pseudorabies virus. J. Virol. 64, 4691-4696.

Favoreel, H.W., Van Minnebruggen, G., Adriaensen, D., Nauwynck, H.J., 2005. Cytoskeletal rearrangements and cell extensions induced by the US3 kinase of an alphaherpesvirus are associated with enhanced spread. Proc. Natl. Acad. Sci. U.S.A., Published June 10th, 2005 on the PNAS Early Edition website (e-pub ahead of print).
Geenen, K., Favoreel, H.W., Olsen, L., Enquist, L.W., Nauwynck, H.J., 2005. The pseudorabies virus US3 protein kinase possesses anti-apoptotic activity that protects cells from apoptosis during infection and after treatment with sorbitol or staurosporine. Virology 331, 144-150.

Harada, H., Becknell, B., Wilm, M., Mann, M., Huang, L.J., Taylor, S.S., Scott, J.D., Korsmeyer, S.J., 1999. Phosphorylation and inactivation of BAD by mitochondria-anchored protein kinase A. Mol. Cell. 3, 413-422.

Kato, A., Yamamoto, M., Ohno, T., Kodaira, H., Nishiyama, Y., Kawaguchi, Y., 2005. Identification of proteins phosphorylated directly by the Us3 protein kinase encoded by herpes simplex virus 1. J. Virol. 79, 9325-9331.

Klupp, B.G., Granzow, H., Mettenleiter, T.C., 2001. Effect of the pseudorabies virus US3 protein on nuclear membrane localization of the UL34 protein and virus egress from the nucleus. J. Gen. Virol. 82, 2363-2371.

Leopardi, R., Van Sant, C., Roizman, B., 1997. The herpes simplex virus 1 protein kinase US3 is required for protection from apoptosis induced by the virus. Proc. Natl. Acad. Sci. U.S.A. 94, 7891-7896.

Leung-Tack, P., Audonnet, J.C., Riviere, M., 1994. The complete DNA sequence and the genetic organization of the short unique region (US) of the bovine herpesvirus type 1 (ST strain). Virology 199, 409-421.

Murata, T., Goshima, F., Daikoku, T., Takakuwa, H., Nishiyama, Y., 2000. Expression of herpes simplex virus type 2 US3 affects the Cdc42/Rac pathway and attenuates c-Jun N-terminal kinase activation. Genes Cells 5, 1017-1027.

Ogg, P.D., McDonell, P.J., Ryckman, B.J., Knudson, C.M., Roller, R.J., 2004 The HSV-1 Us3 protein kinase is sufficient to block apoptosis induced by overexpression of a variety of Bcl-2 family members. Virology 319 , 212-224.

Piroozmand, A., Koyama, A.H., Shimada, Y., Fujita, M., Arakawa, T., Adachi, A., 2004. Role of Us3 gene of herpes simplex virus type 1 for resistance to interferon. Int. J. Mol. Med. 14, 641-645.

Poon, A.P., Benetti, L., Roizman, B., 2006. U(S)3 and U(S)3.5 protein kinases of herpes simplex virus 1 differ with respect to their functions in blocking apoptosis and in virion maturation and egress. J. Virol. 80, 37523764.

Reynolds, A.E., Wills, E.G., Roller, R.J., Ryckman, B.J., Baines, J.D., 2002. Ultrastructural localization of the herpes simplex virus type 1 UL31, UL34, and US3 proteins suggests specific roles in primary envelopment and egress of nucleocapsids. J. Virol. 76, 8939-8952.

Ryckman, B.J., Roller, R.J., 2004. Herpes simplex virus type 1 primary envelopment: UL34 protein modification and the US3-UL34 catalytic relationship. J. Virol. 78, 399-412.

Schaap, A., Fortin, J., Sommer, M., Zerboni, L., Stamatis, S., Ku, C., Nolan, G.P., Arvin, A.M., 2005. T-Cell tropism and the role of ORF66 protein in pathogenesis of varicella-zoster virus infection. J. Virol. 79, 12921-12933.

Schumacher, D., Tischer, B.K., Trapp, S., Osterrieder, N., 2005. The protein encoded by the US3 orthologue of Marek's disease virus is required for efficient de-envelopment of perinuclear virions and involved in actin stress fiber breakdown. J. Virol. 79, 3987-3997.

Sloan, D.D., Zahariadis, G., Posavad, C.M., Pate, N.T., Kussick, S.J., Jerome, K.R., 2003. CTL are inactivated by herpes simplex virus-infected cells expressing a viral protein kinase. J. Immunol. 171, 6733-6741.

Van Minnebruggen, G., Favoreel, H.W., Jacobs, L., Nauwynck, H.J., 2003. Pseudorabies virus US3 protein kinase mediates actin stress fiber breakdown. J. Virol. 77, 9074-9080.

van Zijl, M., Quint, W., Briaire, J., De Rover, T., Gielkens, A., Berns, A., 1988. Regeneration of herpesviruses from molecularly cloned subgenomic fragments. J. Virol. 62, 2191-2195.

van Zijl, M., van der Gulden, H., de Wind, N., Gielkens, A., Berns, A., 1990. Identification of two genes in the unique short region of pseudorabies virus: comparison with herpes simplex virus and varicella-zoster virus. J. Gen. Virol. 71, 1747-1755.

Wagenaar, F., Pol, J.M., Peeters, B., Gielkens, A.L., de Wind, N., Kimman, T.G., 1995. The US3-encoded protein kinase from pseudorabies virus affects egress of virions from the nucleus. J. Gen. Virol. 76, 1851-1859. 